\title{
Ability and Stability of Running and Walking in Children with Cerebral Palsy
}

\author{
Marco losa ${ }^{1}$ Daniela Morelli ${ }^{2}$ Tiziana Marro ${ }^{2}$ Stefano Paolucci, ${ }^{1,2}$ Augusto Fusco ${ }^{1}$ \\ ${ }^{1}$ Clinical Laboratory of Experimental Neurorehabilitation, Fondazione \\ Santa Lucia IRCCS, Rome, Italy \\ 2 Department of Neurorehabilitation, Fondazione Santa Lucia IRCCS, \\ Address for correspondence Marco losa, PhD, Clinical Laboratory of \\ Experimental Neurorehabilitation, Fondazione Santa Lucia IRCCS, \\ Rome, Italy \\ Via Ardeatina 306, 00179 Rome, Italy (e-mail: m.iosa@hsantalucia.it).
}

Neuropediatrics 2013;44:147-154.

\begin{abstract}
Keywords

- cerebral palsy

- gait

- kinematics

- accelerometry

- sport

Objectives Many studies have examined how children with cerebral palsy (CP) manage to walk, but few have investigated running, yielding controversial results. The aim of this study was to quantitatively assess gait ability and its stability in children with hemiplegic $\mathrm{CP}$ while running and walking.

Methods A group of 20 children with spastic hemiplegia due to CP (CPG, $5.1 \pm 2.3$ years old), and a group of 20 children with typical development (TDG, $5.9 \pm 2.6$ years old) underwent a 10-m walking/running test with a wearable triaxial accelerometer fixed to their lower trunk. Spatiotemporal gait parameters, root mean squares of upper body acceleration, and related harmonic and symmetry ratios were computed.

Results Differences in gait speed were significantly higher during running ( $-19 \%$ for CPG with respect of TDG) than during walking ( $-14 \%, p=0.028)$. Conversely, no significant changes were observed in terms of gait stability, and the differences in terms of gait harmony along anteroposterior axis recorded during walking $(-43 \%, p<0.001)$ disappeared during running $(+3 \%, p=0.834)$.

Conclusions During running, children with $\mathrm{CP}$ are slower than children with TD, but their gait was not less stable, and the harmony of their anteroposterior movements was even more similar to TDG than during walking.
\end{abstract}

\section{Introduction}

Cerebral palsy (CP) is the most common cause of physical disability in childhood. ${ }^{1}$ It is a neurodevelopmental disorder caused by nonprogressive lesions in the immature brain causing sensorimotor and cognitive impairments. ${ }^{2}$ From a motor point of view, it can manifest itself in hemi-, di-, or tetraparesis, implying the failure to acquire physiologic locomotor schemas. ${ }^{1}$ Nevertheless, approximately two-thirds of children with cerebral palsy manage to walk at age 5 years. ${ }^{1}$ Atypical, but functional, locomotor patterns can emerge in mildly to moderately affected children. ${ }^{3}$ Although children with hemiparesis have motor asymmetries, research has demonstrated that they benefit from their intrinsically stiffer system by transferring energy from the nonaffected to the affected lower extremity, ${ }^{3}$ similarly to the energy use in physiologic running gaits. ${ }^{4}$

Many studies have analyzed walking in children with CP, but few have examined running. Running, when possible, is a common type of locomotion in children, notwithstanding any diseases. Moreover, there is a significant controversy in the literature: Whereas some groups observed deterioration of locomotor patterns during running ${ }^{5}$ and discouraged physical activities to decrease the risk of spasticity, ${ }^{6}$ others reported more physiologic kinematic profiles in children with $\mathrm{CP}$ during running ${ }^{7}$ and demonstrated positive effects of physical training on physical, psychological, and social wellbeing without adversely affecting spasticity or movement patterns. $^{8-10}$ received

July 17, 2012

accepted after revision

January 7, 2013

published online

March 13, 2013
C 2013 Georg Thieme Verlag KG Stuttgart · New York
DOI http://dx.doi.org/ 10.1055/s-0033-1336016. ISSN $0174-304 X$. 
Running has also been suggested as a movement that should be investigated, having important clinical information. ${ }^{11,12}$ Also, the Gross Motor Classification Measure includes in the locomotor domain (subscore E) walking, running, and jumping. ${ }^{13}$ Thus, greater attention should be paid to the second most common type of locomotion in children and on its relationship to walking.

Although gait lower limb asymmetries can deteriorate during running in children with $\mathrm{CP},{ }^{5}$ these asymmetries do not necessarily result in whole-body asymmetric movements. In fact, children with $\mathrm{CP}$ can counteract the substantial rises in angular momentum generated by asymmetric lower limb patterns by increasing upper limb swing on the unaffected side, ${ }^{11}$ especially during quick walking. ${ }^{14}$ Despite their compensations strategies, children with $\mathrm{CP}$ are less able than children with typical growth to develop a functional and stable gait that manages the emerging instabilities related to the progressive increase in walking speed during development. $^{15}$

Gait stability can be defined as the capacity to minimize upper body oscillations and absorb jerks, bumps, shakes, and fluctuations, despite the broad and fast movements of the lower limbs during locomotion. ${ }^{16}$ Hence, an upright gait is stable when upper body accelerations are minimized and smoothed and have a harmonic distribution that is in accordance with natural step-by-step repetition. ${ }^{17}$ The stability of gait can hence be assessed evaluating symmetry, ${ }^{18,19}$ smoothness, ${ }^{16}$ and harmony ${ }^{15}$ of the locomotor act. All these features can be quantitatively assessed by measuring upper body accelerations. ${ }^{15,19}$

Although gait stability has already been assessed in children with $\mathrm{CP}$ during walking, ${ }^{15}$ no controlled studies have compared gait stability between children with $\mathrm{CP}$ and those with typical development (TD) during running. The lack of studies on running in $\mathrm{CP}$ together with a common conservative approach, mainly based on Bobath rehabilitative concepts, ${ }^{6}$ may have discouraged running and sport activities in children with CP. This approach was also supported by some studies showing at faster gait speeds an increment of spasticity in hamstrings and calf muscles ${ }^{20-22}$ and deteriorating gait asymmetries with running. ${ }^{5}$ Moreover, a poor active life in children with $\mathrm{CP}$ is also due to inadequate structures and qualified staff to allow them to participate in sports and afterschool activities. ${ }^{23}$ Many factors, including the parental level of education, contribute to an inadequate level of physical activity in about $90 \%$ of children with $\mathrm{CP} .{ }^{10}$ An insufficient level of physical activity may contribute to the development of secondary symptoms associated with CP, such as loss of functional abilities, fatigue, osteoporosis, and, for young children, also an inadequate development of the cardiorespiratory system. ${ }^{10}$ In this scenario, clinicians may not feel comfortable encouraging sport activities in these children because biomechanical data about running patterns in children with $\mathrm{CP}$ is still controversial. ${ }^{5,7}$

The aim of our study was to examine the ability and stability of running and walking in mildly affected children with hemiplegia due to $\mathrm{CP}$ versus an age-matched group of children with TD. According to the objective of this research, we tested the following two hypotheses:

1. Gait stability of children with $\mathrm{CP}$ is more affected than that of children with TD during running than during walking.

2. Children having higher instabilities during walking are also those less stable during running, with walking instabilities deteriorated during running.

\section{Patients and Methods}

To address the above issues, we performed an analysis investigating if the differences between a group of children with hemiplegic cerebral palsy (CPG) and an age-matched group of children with typical development (TDG) increased during running with respect to walking. Then, to verify if these differences are related to the locomotor patterns common to the two types of gait, we performed a within-subject analysis correlating the parameters evaluated in both the locomotor conditions. Because within-subject analysis can be inflated by age (for antropometric measurements and motor development), we corrected this last analysis for the age of each child (see Statistical Analysis, below).

\section{Participants}

Twenty children with a clinical diagnosis of spastic hemiplegia due to $\mathrm{CP}$ (CPG: 11 boys and 9 girls; mean age: $5.1 \pm 2.3$ years; mean height: $1.10 \pm 0.17 \mathrm{~m} ; 14$ right and 6 left hemiplegia) and a group of 20 children with TD (TDG: 13 boys and 7 girls; matched with CPG for age: $5.9 \pm 2.7$ years, $p=0.298$, and height: $1.20 \pm 0.20 \mathrm{~m}, p=0.106$ ) were enrolled in this study. Our local ethics committee approved the study procedures that adhered to the Declaration of Helsinki for medical research involving human subjects, and informed consent was obtained from both parents of each child. Because the definition of $\mathrm{CP}$ is usually not etiologic (CP causes) but functional (how it manifests itself), ${ }^{2}$ the inclusion criteria of hemiplegia in this study was also based on the assessment of motor functioning. Accordingly, hemiplegia has been defined as a neuromuscular disorder that involves one-half of the body in the frontal plane while the other half is normal (14 of 20 children in our study) or near normal ( 6 children). ${ }^{24}$

All participants were able to walk and run in an autonomous manner for at least $10 \mathrm{~m}$, to understand the instructions, and to perform the test properly. Most children with CP had a loss of periventricular white matter ( 14 of 18 children having available magnetic resonance images), usually accompanied by different lesions in other brain areas specific for each child, which was in accordance with prevalence and etiology of $\mathrm{CP}^{25}$ They took their first steps at the age of $20 \pm 6$ months. All children with $\mathrm{CP}$ were classified by rehabilitative staff between level I and II of the Gross Motor Function Classification System. ${ }^{26}$ Their motor abilities were assessed by Gross Motor Function Measure (GMFM-88), a standardized observational instrument of 88 items grouped into 5 domains, related primarily to postural and locomotor abilities. $^{13}$ Their mean GMFM-88 score was $88.3 \pm 8.6 \%$ and the mean subscore-E (walking, running, and jumping) was $71.9 \pm 15.8 \%$. Their gait patterns were also visually evaluated 
by the rehabilitative staff according to Winters and Gage classification based on four levels of lower limb impairment during gait ${ }^{24}$ : 11 children had an ankle plantar-flexion in swing phase (1); 5 children had a static or dynamic contracture of the gastrocnemius and soleus muscles resulting in persistent plantar flexion of the ankle during the stance and swing phases (2); 4 children showed a hyperactivity of quadriceps and hamstrings limiting flexion of the knee (3); and no child had a reduction of motion in the sagittal plane at the hip (4).

\section{Protocol}

Children were asked to stand still on a line on the floor and walk or run straight for $10 \mathrm{~m}$ at a self-selected speed until arriving at another line on the floor in a 30-m-long rehabilitation gym. They wore an elastic belt with a wearable inertial sensor device (FreeSense, Sensorize s.r.l., Rome, Italy; sampling frequency $=100 \mathrm{~Hz}, \pm 58.8 \mathrm{~m} / \mathrm{s}^{2}$ of acceleration full range, weight $93 \mathrm{~g}$, height $8.8 \mathrm{~cm}$, width $5.1 \mathrm{~cm}$, depth $2.5 \mathrm{~cm}$ ) on an area of their back that corresponded to the L2-L3 spinous processes, close to their center of mass. This device contains a triaxial accelerometer to measure accelerations along the three body axes (anteroposterior, laterolateral, and craniocaudal axis). Data capture was managed using a Bluetooth protocol and directly loaded into a database. A similar experimental setup was already used in a previous study on walking stability in children with $\mathrm{CP} .{ }^{15}$ The accelerometric data were shown to be reliable and valid for the gait assessment in healthy subjects ${ }^{27}$ and patients with stroke. ${ }^{17}$ This method of measurement has been validated also for running and hopping in children with TD (age range: 2 to 12 and 3 to 12 years, respectively). ${ }^{28,29}$ An accuracy of $87 \%$ was achieved for accelerometric data in the discrimination between high and low developmental level from healthy children during running task. 28

During the test, all subjects wore their commonly used shoes without aids or orthoses. Children of both groups performed each test after a short familiarization period (about one or two trials for walking and one or two for running), and the following experimental trial was repeated if the child did not follow a straight trajectory or stopped his or her movement during the test (a second walking trial was needed in 8 of 20 children from CPG and 5 TDG; a third in 1 child with $\mathrm{CP}$; a second running trial in 7 children from CPG and 2 TDG; and a third running trial in 1 child from CPG and 1 TDG).

Similarly to previous research, ${ }^{15}$ the acceleration data were recorded during eight consecutive steps (four strides) taken in the central part of the walking pathway and were analyzed after their mean subtraction and 20-Hz low-pass filtering. Each contact with the foot (beginning of a step) was identified by minimum of anteroposterior acceleration (-Fig. 1 ). Three parameters for locomotor ability and three for locomotor stability were analyzed:

1. Locomotor ability:

- Gait speed was measured as $10 \mathrm{~m}$ per time spent to complete the pathway.
- Step length was estimated as 10 m per number of steps.

- Step duration was computed as the mean of eight values obtained by timing of the anteroposterior acceleration minima.

2. Locomotor stability:

- Gait smoothness: Mean root mean square (RMS) of accelerations measured by the wearable device placed close to the body center of mass, which is a measure of instability and coincides with the standard deviation of the acceleration signal due to signal mean subtraction; the mean of four values (one for each analyzed stride) was computed along each body axis and then averaged among these axes. ${ }^{27}$

- Gait harmony: Mean of accelerometric harmonic ratio (HR), which is an indicator of the step-by-step rhythmicity of acceleration patterns, computed as the mean of four values (one for each analyzed stride), evaluated as the ratio between the sum of even/odd for anteroposterior and craniocaudal or odd/even for laterolateral harmonic amplitudes, calculated by discrete Fourier transform, and averaged among the three body axes. ${ }^{27}$

- Gait symmetry: Mean of ratio index (RI) that is a normalized indicator of asymmetry and is computed as the percentage ratio between the lower/higher absolute values of the means of minimum acceleration for the right and left legs (mean values were computed for four steps each along each body axis and then averaged among the three body axes). ${ }^{30}$

\section{Statistical Analysis}

The mean \pm standard deviation was computed for all parameters. To test if children with $\mathrm{CP}$ were less stable than those with TD during running with respect of walking, a $2 \times 2$ mixed analysis of variance was performed using as independent variables the group (between-subject factor: CPG versus TDG) and type of locomotion (within-subject factor: running versus walking) and including the interaction of these two factors into the model (group $\times$ locomotion). Statistically significant interactions were followed by post-hoc comparisons. The effect size was estimated using partial eta squared, $\boldsymbol{\eta}_{\boldsymbol{p}}{ }^{2}$ (the Cohen thumb rule suggests to consider it small if $<0.06$, medium if between 0.06 and 0.14 , and large if $>0.14$ ).

To test if children having higher instabilities during walking were also those less stable during running, we used Pearson correlation coefficient between parameter values assessed during the two tasks. This hypothesis was implicitly suggested by Böhm and Döderlein, ${ }^{5}$ but that study did not attempt to verify it. In fact, that study did not perform any correlation analysis between walking and running impairments. Because this correlation evaluated between walking and running within each group can be inflated by age (for the anthropometric dimensions and developmental process), a partial correlation coefficient $(R)$ was used to evaluate these correlations controlled for age. ${ }^{15}$ Microsoft Office Excel was used to generate graphs, SPSS 17.0 (SPSS Inc., Chicago, Illinois, United States) was used for all statistical analyses, and the significance level was set at $p<0.05$, with the exception of posthoc comparisons for which Bonferroni correction was applied. 


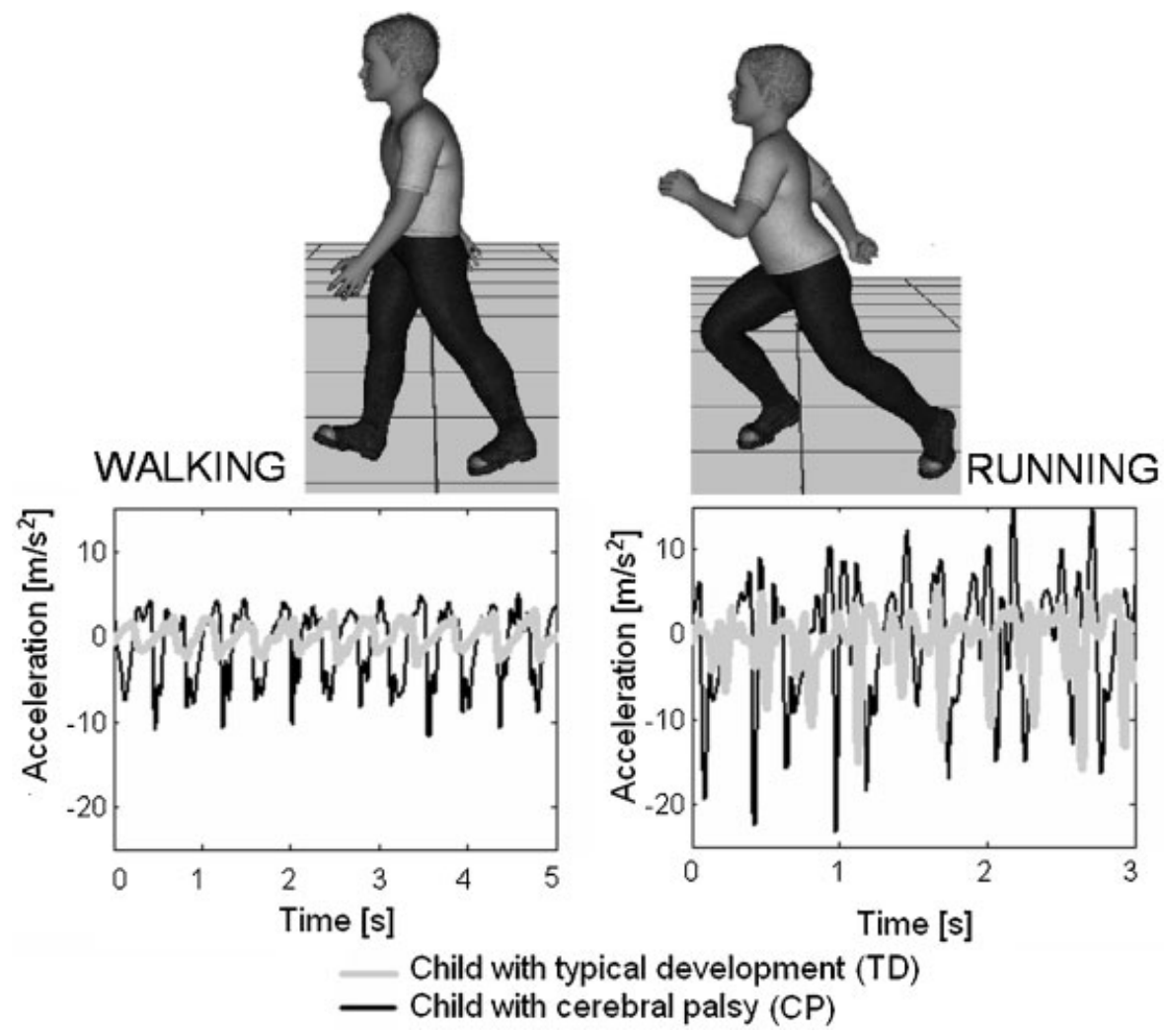

Fig. 1 Acceleration signals along the anteroposterior body axis. Acceleration signals were recorded during walking (on the left) and running (on the right) of a child with cerebral palsy (black lines) and one with typical development (gray lines).

\section{Results}

In both groups of children, the mean speed was more than doubled during running with respect to walking, as a result of an increase in step length and a decrease in step duration (-Fig. 2). - Table 1 shows as the main factor "locomotion" significantly affected all the spatiotemporal gait parameters and the smoothness of upper body accelerations. The main factor "group" affected gait speed and step length but not step duration. The difference between groups significantly increased during running ( $-19 \%$ for CPG vs. TDG) than during walking ( $-14 \%$ ), but only for gait speed, as shown by the significant group $\times$ locomotion interaction (-Table 1, - Fig. 2). Post-hoc analyses reveled a significant difference between CPG and TDG during running ( $p=0.011)$ but not during walking $(p=0.044$, not significant for Bonferroni correction).

The differences between CPG and TDG (group effect) were significant for HR and RI, whereas type of locomotion only affected the acceleration RMS in both groups. No significant interactions between locomotor type and group factors were noted for any parameter related to gait stability, meaning the differences between groups were not significantly changed from walking to running. The interaction effect was close to the significant threshold only for HR $(p=0.083)$ but revealing less difference in running $(-15 \%)$ than in walking $(-35 \%$, see -Fig. 2). Because of this $p$ value close to significant threshold, a deeper analysis was performed on the HR, along each one of the three body axes. A highly significant interaction group $\times$ locomotion was found for the anteroposterior

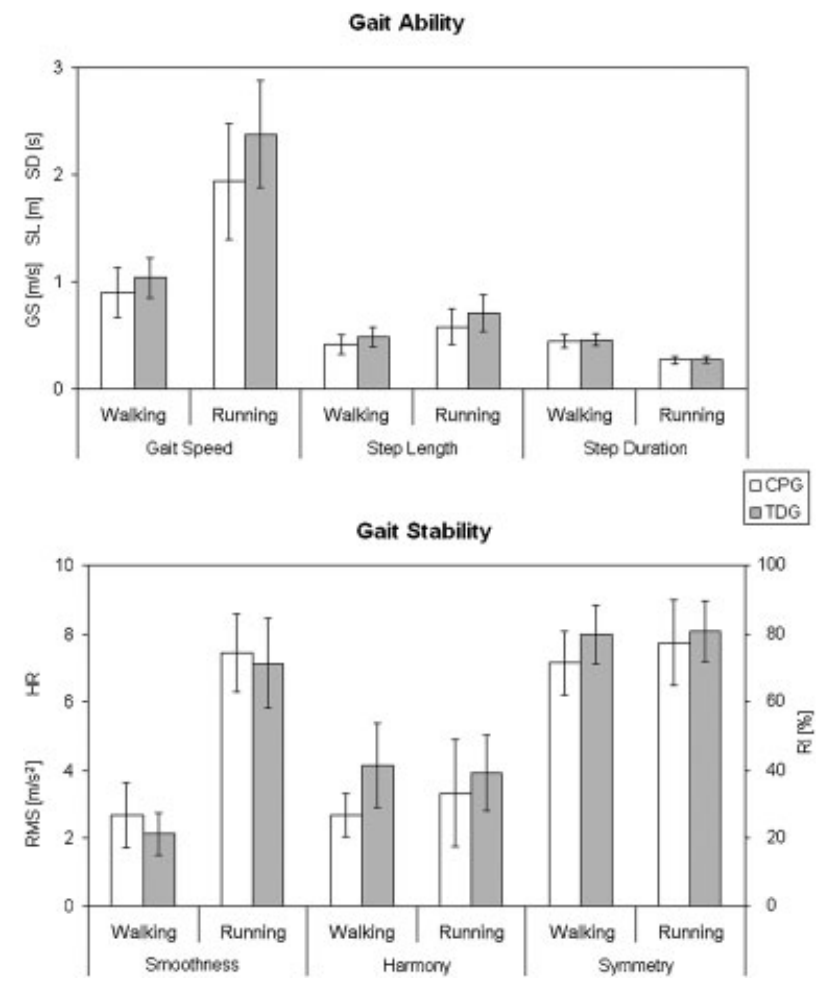

Fig. 2 Gait ability and stability. Mean \pm standard deviation of parameters related to locomotor ability (above: gait speed GS, step length SL, step duration SD) and stability (below: acceleration root mean square RMS, that inversely represent smoothness of movements, harmonic ratio HR and symmetry ratio index $\mathrm{RI}$ ) for children with cerebral palsy (empty bars) and those with typical development (gray bars) during walking and running. 
Table 1 Factors affecting gait ability and stability

\begin{tabular}{|c|c|c|c|c|c|}
\hline & Parameters & & Locomotion & Group & Group $\times$ Locomotion \\
\hline \multirow[t]{3}{*}{ Ability } & Gait speed & GS & $\begin{array}{l}F=326.791 \\
p<0.001 \\
\eta_{p}{ }^{2}=0.896\end{array}$ & $\begin{array}{l}F=7.309 \\
p=0.010 \\
\eta_{p}{ }^{2}=0.161\end{array}$ & $\begin{array}{l}F=5.224 \\
p=0.028 \\
\eta_{p}^{2}=0.121\end{array}$ \\
\hline & Step length & SL & $\begin{array}{l}F=111.487 \\
p<0.001 \\
\eta_{p}^{2}=0.746\end{array}$ & $\begin{array}{l}F=6.217 \\
p=0.017 \\
\eta_{p}{ }^{2}=0.141\end{array}$ & $\begin{array}{l}F=2.279 \\
p=0.139 \\
\eta_{p}^{2}=0.057\end{array}$ \\
\hline & Step duration & SD & $\begin{array}{l}F=489.263 \\
p<0.001 \\
\eta_{p}^{2}=0.928\end{array}$ & $\begin{array}{l}F=0.213 \\
p=0.647 \\
\eta_{p}^{2}=0.006\end{array}$ & $\begin{array}{l}F=0.485 \\
p=0.490 \\
\eta_{p}{ }^{2}=0.013\end{array}$ \\
\hline \multirow[t]{3}{*}{ Stability } & Smoothness & RMS & $\begin{array}{l}F=486.624 \\
p<0.001 \\
\eta_{p}{ }^{2}=0.928\end{array}$ & $\begin{array}{l}F=3.042 \\
p=0.089 \\
\eta_{p}{ }^{2}=0.074\end{array}$ & $\begin{array}{l}F=0.356 \\
p=0.554 \\
\eta_{p}{ }^{2}=0.009\end{array}$ \\
\hline & Harmony & $\mathrm{HR}$ & $\begin{array}{l}F=0.731 \\
p=0.398 \\
\eta_{\mathrm{p}}{ }^{2}=0.019\end{array}$ & $\begin{array}{l}F=13.073 \\
p=0.001 \\
\eta_{p}{ }^{2}=0.256\end{array}$ & $\begin{array}{l}F=3.161 \\
p=0.083 \\
\eta_{\mathrm{p}}{ }^{2}=0.077\end{array}$ \\
\hline & Symmetry & RI & $\begin{array}{l}F=0.275 \\
p=0.105 \\
\eta_{p}^{2}=0.068\end{array}$ & $\begin{array}{l}F=6.126 \\
p=0.018 \\
\eta_{p}^{2}=0.139\end{array}$ & $\begin{array}{l}F=1.382 \\
p=0.247 \\
\eta_{p}^{2}=0.035\end{array}$ \\
\hline
\end{tabular}

Results of mixed ANOVA (in bold if significant, $p<0.05$ ). The degrees of freedom $(F)$ were 1,38 . The partial eta squared $\left(\eta_{p}{ }^{2}\right)$ values are reported as estimation of effect size.

$\operatorname{HR}\left(F_{1,38}=13.373, p=0.001\right)$, whereas this effect was neither significant along laterolateral $(p=0.707)$ nor along craniocaudal axis $(p=0.904)$. Post-hoc analysis revealed that the harmony of movements along the anteroposterior direction was significantly different between the two groups during walking ( $-43 \%$ for CPG with respect to TDG, $p<0.001$ ) but not during running $(+3 \%, p=0.834)$, as depicted in -Fig. 3.

The parameters related to gait ability were not significantly correlated between walking and running in TDG, whereas in CPG the partial correlations controlled for age were (for SD) or were close to being (for gait speed and step length) statistically significant (-Table 2 ). Conversely, neither children with CP nor those with TD had a significant correlation between running and walking parameters related to gait stability.

The most affected child with the lowest walking $(0.57 \mathrm{~m} / \mathrm{s})$ and running $(0.87 \mathrm{~m} / \mathrm{s})$ speeds also had the lowest harmonic

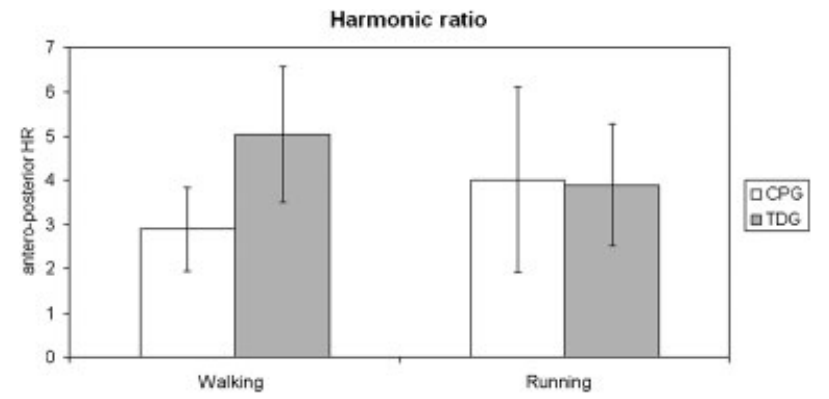

Fig. 3 Harmonic ratio. Mean \pm standard deviation of harmonic ratio evaluated along the anteroposterior body axis for children with cerebral palsy (empty bars) and those with typical development (gray bars) during walking and running. ratio during both these tasks (1.69 and 1.42, respectively), despite a level 1 in Winters and Gage classification. From a neurologic point of view, she was the only participant with a lesion in the internal capsule (together with a lesion in corona radiata). HR was also found to be low in a child with loss of periventricular white matter and a lesion in the basal ganglia, in a child with parenchymal loss of white matter, and in the only two children with no available magnetic resonance image. Two other children had a lesion involving the basal ganglia: one had a low HR during running (2.12) but not during walking (3.48), whereas the other had a high HR during running (5.74) and an adequate HR during walking (2.68). Finally, the less stable running patterns (RMS $=9.37$ $\mathrm{m} / \mathrm{s}^{2}$ ) were observed in a child with a brain lesion in corona

Table 2 Relationship between running and walking

\begin{tabular}{|c|c|c|c|c|}
\hline & \multicolumn{2}{|c|}{ Parameters } & CPG & TDG \\
\hline \multirow[t]{3}{*}{ Ability } & Gait speed & GS & $\begin{array}{l}R=0.446 \\
p=0.056\end{array}$ & $\begin{array}{l}R=0.364 \\
p=0.125\end{array}$ \\
\hline & Step length & SL & $\begin{array}{l}R=0.442 \\
p=0.058\end{array}$ & $\begin{array}{l}R=0.151 \\
p=0.536\end{array}$ \\
\hline & Step duration & SD & $\begin{array}{l}R=0.478 \\
P=0.038\end{array}$ & $\begin{array}{l}R=0.299 \\
p=0.214\end{array}$ \\
\hline \multirow[t]{3}{*}{ Stability } & Smoothness & RMS & $\begin{array}{l}R=0.049 \\
p=0.852\end{array}$ & $\begin{array}{l}R=0.136 \\
p=0.579\end{array}$ \\
\hline & Harmony & $\mathrm{HR}$ & $\begin{array}{l}R=0.188 \\
p=0.441\end{array}$ & $\begin{array}{l}R=0.211 \\
p=0.385\end{array}$ \\
\hline & Symmetry & RI & $\begin{array}{l}R=0.227 \\
p=0.350\end{array}$ & $\begin{array}{l}R=-0.018 \\
p=0.941\end{array}$ \\
\hline
\end{tabular}

Partial correlation coefficient $(R)$ controlled for age (in bold if significant, $p<0.05)$. 
radiata and centrum semiovale, together with loss of periventricular white matter. Conversely, he showed an adequate upright stability during walking (RMS $=2.62 \mathrm{~m} / \mathrm{s}^{2}$ ).

\section{Discussion}

The aim of this study was to investigate the differences in terms of gait ability and stability between children with CP and those with TD during running and walking. With regard to the main effects, we found that group, and hence CP, significantly affected gait speed, step length, harmony, and symmetry of movements. These results were in accordance to previous studies on walking ${ }^{31}$ and running. ${ }^{7}$ Furthermore, according with literature, ${ }^{7}$ we found that type of locomotion significantly affected gait speed, step length, and step duration in both groups. Type of locomotion also affected the smoothness of lower trunk accelerations. Unexpectedly, we found that the interaction of group $\times$ locomotion affected only gait speed, revealing that the differences between children with $\mathrm{CP}$ and those with TD did not change in terms of gait stability during running with respect to walking. Hence, we failed to find significant results supporting the hypothesis that gait stability of children with $\mathrm{CP}$ was more affected than that of children with TD during running than during walking.

Despite the differences between studies, Böhm and Döderlein, analyzing the symmetry of lower limb kinematics, also noted the main effects of locomotion and pathology, but not of their interaction. The higher asymmetries observed during running compared with walking led them to conclude that the walking asymmetries deteriorate during running. ${ }^{5}$ However, they did not normalize their raw values for the increased joint range of motion recorded during running. Analyzing accelerations and normalizing values, we failed to note any significant deterioration of gait asymmetries during running with respect to walking. These differences must be interpreted with caution due to the methodologic differences between the study of Böhm and Döderlein, ${ }^{5}$ in which a stereophotogrammetric system was used for measuring lower limb joint angles, and our study, in which a wearable device was used for measuring upper body accelerations. However, in that study no attempt was made to quantify the association between walking and running asymmetries and whether the same asymmetric patterns are involved in both locomotor acts. In our study, we failed to find significant correlations between running and walking values in terms of gait stability. The absence of these correlations suggested that $\mathrm{CP}$ affected in different ways walking and running stability of each child. Therefore, we rejected the second hypothesis of this study. Few correlations were found in terms of spatiotemporal parameters: They might be strictly related to body impairments rather than to the acquisition of proper locomotor patterns in children with $\mathrm{CP}$. These results may suggest that a child with CP may achieve a different level of ability in the execution of different locomotor tasks. Despite the severity of $\mathrm{CP}$ and the possible limitations in joint ranges of motion, our results suggest that it is not straightforward that the most severely impaired children during walking should be the most impaired during running as well. Eek and colleagues found that, after a muscle strength training, gross motor functions of trained children improved despite walking speed remaining unchanged. ${ }^{32}$

From a neurologic point of view, we found that a reduction of walking and running harmonic ratio was observed in one child with a lesion in internal capsule and in two of three children with a lesion involving the basal ganglia. It has already been reported that an affected development of the internal capsule is related to the severity of gait and motor deficits. $^{33}$ The involvement of basal ganglia may affect the conveying of locomotor signals from frontal cortex to the centers for locomotion and postural control in the brainstem tegmentum. ${ }^{34}$

Different activations have been observed in some brain areas during the execution of a running task with respect to walking. In particular, higher cerebellar activation in the vermis and adjacent hemispheres and a deactivation in the vestibular and somatosensory cortex were found during running than during walking. ${ }^{35}$ These different activities may be at the basis of our results showing that running stability was not significantly correlated to walking stability.

In fact, gait smoothness (acceleration RMS) was not affected by the interaction between group and locomotion type, revealing that instabilities increased during running, but similarly for both groups. However, it should be noted that acceleration RMS are strictly related to gait speed, ${ }^{15,17}$ and we found similar acceleration values despite the different speeds of the two groups of subjects. It is possible that both groups run with the maximum accelerations they were able to manage, and these accelerations were similar between children with $\mathrm{CP}$ and those with TD, despite resulting in a higher speed for TDG. This possible interpretation seems to be in accordance with previous studies reporting as the running of children with $\mathrm{CP}$ is poorly efficient, despite the more physiologic kinematic profiles in sagittal plane during running rather than in walking., ${ }^{5,7}$ Also in our study we found more physiologic step-by-step rhythmicity (higher harmonic ratio) during running of children with CP. Davids et al had already suggested that children with $\mathrm{CP}$ assume a gait posture or profile that may impair walking yet facilitate running, such as toe strike at initial contact and increased plantar flexion throughout stance phase. ${ }^{7}$ Furthermore, during running, the knee and hip are more flexed than in walking, the pelvis and trunk are tilted forward, ${ }^{36}$ and the activity of arm flexor muscles and leg extensor muscles is higher and prolonged. ${ }^{37}$ These patterns are often observed during walking for the hemiplegic side of the body in children with CP and related to their spasticity. ${ }^{31}$ Thus, these patterns may increase the gait harmony, because they are likely more functional or at least more tolerated during running, when also the contralateral lower limb joints are more flexed. ${ }^{7}$ This bent-hip, bent-knee gait with the trunk sloping forward and more plantar-flexed ankle, typical of running, has some similarities with the ancestral bipedal gait. ${ }^{38}$ It has been suggested that locomotion is built starting from common primitives related to an ancestral neural network ${ }^{39}$ and locomotor primitives are invariantly present in a variety of locomotion conditions, including walking and running. ${ }^{40,41}$ In our study we did not 
find significant correlations between walking and running parameters, revealing that a child with a functional walk can have an instable run or vice versa. It was, for example, the case of a child with a lesion in the corona radiata and centrum semiovale who showed a good walking stability, but with the lowest upright stability during running. This seems to be in contrast with the idea that walking patterns deteriorate during running. Despite these differences, specific brain lesions, such as of the internal capsule or basal ganglia, may increase motor deficits, ${ }^{33}$ affecting both the locomotor types.

However, we did not perform electromyographic measurements to deeply investigate muscular activities and locomotor primitives, and it is the chief limitation of our study. Further research using electromyography should address three main issues: if running and walking and if running and spasticity have common patterns and if running training may increase spasticity. That spasticity is velocity dependent ${ }^{42}$ and that running and spasticity may have common patterns are two possible explanations for the fear that physical training and sports can increase spasticity. ${ }^{43}$ Yet, studies exist on the positive effects of sport and physical activities on functional outcomes and quality of life of people with CP and their families. ${ }^{9,10,44,45}$

Another limitation of our study is the reduced sample sizes of the two groups of children. Furthermore, we investigated the kinematics of a point close to the center of mass similarly to previous research on children with $\mathrm{CP}^{15}$ or adults with other pathologies. ${ }^{17,19}$ The use of only one device allowed a simple noninvasive setup but hid the information about the contribution of lower and upper limbs and of paretic and nonparetic sides. Finally, we took into account the side where the brain lesion resided but not its timing because sometimes it was difficult to clearly date the injury, especially if a pre- or perinatal event was not the evident cause. All these limitations suggest caution in the interpretation of our data. Nevertheless, our study has shown that despite increasing speed differences, the stability of running movements are neither inferior and even nor related to that of walking ones. On the contrary, running patterns seemed to be more harmonic than walking patterns along the main direction of locomotion. However, further studies are needed to investigate if spastic and running patterns may be related to some common, and maybe ancestral, locomotor patterns.

\section{Acknowledgments}

This study was supported by our Foundation and by the provincial government "Provincia di Roma" by means of CARESS (from Childhood to Adulthood: Rehabilitation and Enabling Sport for Sociability) and SPORT-AB projects. We thank Patrizia Prestipino and Antonio Rosati for their support in these projects.

\section{References}

1 Krägeloh-Mann I, Cans C. Cerebral palsy update. Brain Dev 2009; 31(7):537-544

2 Bax M, Goldstein M, Rosenbaum P, et al; Executive Committee for the Definition of Cerebral Palsy. Proposed definition and classifi- cation of cerebral palsy, April 2005. Dev Med Child Neurol 2005;47 (8):571-576

3 Fonseca ST, Holt KG, Fetters L, Saltzman E. Dynamic resources used in ambulation by children with spastic hemiplegic cerebral palsy: relationship to kinematics, energetics, and asymmetries. Phys Ther 2004;84(4):344-354, discussion 355-358

4 McMahon TA, Valiant G, Frederick EC. Groucho running. J Appl Physiol 1987;62(6):2326-2337

5 Böhm H, Döderlein L. Gait asymmetries in children with cerebral palsy: do they deteriorate with running? Gait Posture 2012;35 (2):322-327

6 Bobath K. A neurological basis for the treatment of cerebral palsy. 2nd ed. London: William Heineman Books Ltd.; 1980

7 Davids JR, Bagley AM, Bryan M. Kinematic and kinetic analysis of running in children with cerebral palsy. Dev Med Child Neurol 1998;40(8):528-535

8 Fowler EG, Ho TW, Nwigwe AI, Dorey FJ. The effect of quadriceps femoris muscle strengthening exercises on spasticity in children with cerebral palsy. Phys Ther 2001;81(6):1215-1223

9 Fowler EG, Kolobe TH, Damiano DL, et al; Section on Pediatrics Research Summit Participants; Section on Pediatrics Research Committee Task Force. Promotion of physical fitness and prevention of secondary conditions for children with cerebral palsy: section on pediatrics research summit proceedings. Phys Ther 2007;87(11):1495-1510

10 Zwier JN, van Schie PE, Becher JG, Smits DW, Gorter JW, Dallmeijer AJ. Physical activity in young children with cerebral palsy. Disabil Rehabil 2010;32(18):1501-1508

11 Bruijn SM, Meyns P, Jonkers I, Kaat D, Duysens J. Control of angular momentum during walking in children with cerebral palsy. Res Dev Disabil 2011;32(6):2860-2866

12 Verschuren O, Ketelaar M, Gorter JW, Helders PJ, Takken T. Relation between physical fitness and gross motor capacity in children and adolescents with cerebral palsy. Dev Med Child Neurol 2009;51 (11):866-871

13 Russell DJ, Rosenbaum PL, Cadman DT, Gowland C, Hardy S, Jarvis $\mathrm{S}$. The gross motor function measure: a means to evaluate the effects of physical therapy. Dev Med Child Neurol 1989;31(3):341352

14 Meyns P, Van Gestel L, Massaad F, Desloovere K, Molenaers G, Duysens J. Arm swing during walking at different speeds in children with cerebral palsy and typically developing children. Res Dev Disabil 2011;32(5):1957-1964

15 Iosa M, MarroT, Paolucci S, Morelli D. Stability and harmony of gait in children with cerebral palsy. Res Dev Disabil 2012;33(1): 129-135

16 Cappozzo A. Low frequency self-generated vibration during ambulation in normal men. J Biomech 1982;15(8):599-609

17 Iosa M, Fusco A, Morone G, et al. Assessment of upper-body dynamic stability during walking in patients with subacute stroke. J Rehabil Res Dev 2012;49(3):439-450

18 Novak P, Novak V. Effect of step-synchronized vibration stimulation of soles on gait in Parkinson's disease: a pilot study. J Neuroeng Rehabil 2006;3:9

19 Tura A, Raggi M, Rocchi L, Cutti AG, Chiari L. Gait symmetry and regularity in transfemoral amputees assessed by trunk accelerations. J Neuroeng Rehabil 2010;7:4

20 van der Krogt MM, Doorenbosch CA, Harlaar J. The effect of walking speed on hamstrings length and lengthening velocity in children with spastic cerebral palsy. Gait Posture 2009;29(4): 640-644

21 van der Krogt MM, Doorenbosch CA, Becher JG, Harlaar J. Walking speed modifies spasticity effects in gastrocnemius and soleus in cerebral palsy gait. Clin Biomech (Bristol, Avon) 2009;24(5): 422-428

22 van der Krogt MM, Doorenbosch CA, Becher JG, Harlaar J. Dynamic spasticity of plantar flexor muscles in cerebral palsy gait. J Rehabil Med 2010;42(7):656-663 
23 Vogts N, Mackey AH, Ameratunga S, Stott NS. Parent-perceived barriers to participation in children and adolescents with cerebral palsy. J Paediatr Child Health 2010;46(11):680-685

24 Winters TF Jr, Gage JR, Hicks R. Gait patterns in spastic hemiplegia in children and young adults. J Bone Joint Surg Am 1987;69(3):437-441

25 Roelants-van Rijn AM, Groenendaal F, Beek FJA, Eken P, van Haastert IC, de Vries LS. Parenchymal brain injury in the preterm infant: comparison of cranial ultrasound, MRI and neurodevelopmental outcome. Neuropediatrics 2001;32(2):80-89

26 Palisano RJ, Rosenbaum P, Bartlett D, Livingston MH. Content validity of the expanded and revised Gross Motor Function Classification System. Dev Med Child Neurol 2008;50(10):744-750

27 Menz HB, Lord SR, Fitzpatrick RC. Acceleration patterns of the head and pelvis when walking on level and irregular surfaces. Gait Posture 2003;18(1):35-46

28 Masci I, Vannozzi G, Bergamini E, Pesce C, Getchell N, Cappozzo A. Assessing locomotor skills development in childhood using wearable inertial sensor devices: the running paradigm. Gait Posture 2012;pii:S0966-6362(12)00363-3

29 Masci I, Vannozzi G, Getchell N, Cappozzo A. Assessing hopping developmental level in childhood using wearable inertial sensor devices. Mot Contr 2012;16(3):317-328

30 Seliktar R, Mizrahi J. Some gait characteristics of below-knee amputees and their reflection on the ground reaction forces Eng Med 1986;15(1):27-34

31 Gage JR, Schwartz MH, Koop SE. The Identification and Treatment of Gait Problems in Cerebral Palsy. London: Mac Keith Press; 2009

32 Eek MN, Tranberg R, Zügner R, Alkema K, Beckung E. Muscle strength training to improve gait function in children with cerebral palsy. Dev Med Child Neurol 2008;50(10):759-764

33 Rose J, Butler EE, Lamont LE, Barnes PD, Atlas SW, Stevenson DK. Neonatal brain structure on MRI and diffusion tensor imaging, sex and neurodevelopment in very-low-birthweight preterm children. Dev Med Child Neurol 2009;51(7):526-535
34 Jahn K. Vertigo and balance in children-diagnostic approach and insights from imaging. Eur J Paediatr Neurol 2011;15(4):289-294

35 Jahn K, Deutschländer A, Stephan T, Strupp M, Wiesmann M, Brandt $\mathrm{T}$. Brain activation patterns during imagined stance and locomotion in functional magnetic resonance imaging. Neuroimage 2004;22(4):1722-1731

36 Novacheck TF. The biomechanics of running. Gait Posture 1998; 7(1):77-95

37 Ounpuu S. The biomechanics of walking and running. Clin Sports Med 1994;13(4):843-863

38 Alexander RM. Bipedal animals, and their differences from humans. J Anat 2004;204(5):321-330

39 Dominici N, Ivanenko YP, Cappellini G, et al. Locomotor primitives in newborn babies and their development. Science 2011;334 (6058):997-999

40 Cappellini G, Ivanenko YP, Poppele RE, Lacquaniti F. Motor patterns in human walking and running. J Neurophysiol 2006;95(6): 3426-3437

41 Ivanenko YP, Cappellini G, Dominici N, Poppele RE, Lacquaniti F. Coordination of locomotion with voluntary movements in humans. J Neurosci 2005;25(31):7238-7253

42 Lance JW. Symposium synopsis. In: Feldman RG, Young RR, Koella WP, eds. Spasticity: Disordered Motor Control. Chicago, IL: Year Book Medical Publishers; 1980:485-494

43 Kloyiam S, Breen S, Jakeman P, Conway J, Hutzler Y. Soccer-specific endurance and running economy in soccer players with cerebral palsy. Adapt Phys Activ Q 2011;28(4):354-367

44 Groff DG, Lundberg NR, Zabriskie RB. Influence of adapted sport on quality of life: perceptions of athletes with cerebral palsy. Disabil Rehabil 2009;31(4):318-326

45 Capio CM, Sit CH, Abernethy B. Fundamental movement skills testing in children with cerebral palsy. Disabil Rehabil 2011;33 (25-26):2519-2528 